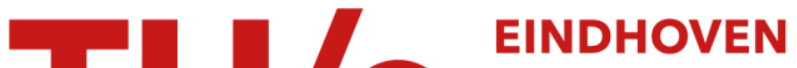 \\ UNIVERSITY OF \\ TECHNOLOGY
}

\section{Velocity trajectory optimization in hybrid electric trucks}

Citation for published version (APA):

Keulen, van, T. A. C., de Jager, B., Foster, D., \& Steinbuch, M. (2010). Velocity trajectory optimization in hybrid electric trucks. In Proceedings of the 2010 American Control Conference (ACC 2010), 30 June 30 - 2 July 2010, Baltimore, Maryland, USA (pp. 5074-5079). Institute of Electrical and Electronics Engineers.

Document status and date:

Published: 01/01/2010

\section{Document Version:}

Publisher's PDF, also known as Version of Record (includes final page, issue and volume numbers)

\section{Please check the document version of this publication:}

- A submitted manuscript is the version of the article upon submission and before peer-review. There can be important differences between the submitted version and the official published version of record. People interested in the research are advised to contact the author for the final version of the publication, or visit the $\mathrm{DOI}$ to the publisher's website.

- The final author version and the galley proof are versions of the publication after peer review.

- The final published version features the final layout of the paper including the volume, issue and page numbers.

Link to publication

\section{General rights}

Copyright and moral rights for the publications made accessible in the public portal are retained by the authors and/or other copyright owners and it is a condition of accessing publications that users recognise and abide by the legal requirements associated with these rights.

- Users may download and print one copy of any publication from the public portal for the purpose of private study or research.

- You may not further distribute the material or use it for any profit-making activity or commercial gain

- You may freely distribute the URL identifying the publication in the public portal.

If the publication is distributed under the terms of Article 25fa of the Dutch Copyright Act, indicated by the "Taverne" license above, please follow below link for the End User Agreement:

www.tue.nl/taverne

Take down policy

If you believe that this document breaches copyright please contact us at:

openaccess@tue.nl

providing details and we will investigate your claim. 


\title{
Velocity Trajectory Optimization in Hybrid Electric Trucks
}

\author{
Thijs van Keulen, Bram de Jager, Darren Foster, and Maarten Steinbuch
}

\begin{abstract}
Hybrid Electric Vehicles (HEVs) enable fuel savings by re-using kinetic and potential energy that was recovered and stored in a battery during braking or driving down hill. Besides, the vehicle itself can be seen as a storage device, where kinetic energy can be stored and retrieved by changing the forward velocity. It is beneficial for fuel consumption to optimize the velocity trajectory in two ways; i) to assist the driver in tracking an optimal velocity trajectory (e.g. input to an Adaptive Cruise Controller); ii) to estimate the future power request trajectory which can be used to optimize the hybrid components use. Taking advantage of satellite navigation, together with the vehicles current mass and road load parameters, an optimization problem is formulated, and solved for a driver defined time constraint. Despite tight velocity constraints, this can result in 5\% fuel saving compared to a Cruise Controller with constant velocity setpoint. The benefit of velocity trajectory optimization is indicated with experimental results.
\end{abstract}

\section{INTRODUCTION}

Hybrid Electric Vehicles (HEVs) enable fuel savings by re-using kinetic and potential energy that was recovered and stored in a battery during braking or driving down hill. Besides the HEV battery, the vehicle itself can be seen as a storage device, where kinetic energy can be stored and retrieved by changing the forward velocity. Therefore, the velocity trajectory can be optimized for certain vehicle operating conditions [1]. The velocity trajectory optimization problem can be defined as: finding a velocity trajectory that minimizes the fuel consumption subject to a time constraint.

In literature several useful contributions can be found regarding velocity trajectory optimization in conventional vehicles. In [2] it is suggested to use Dynamic Programming (DP) to numerically solve the optimal control problem in a hilly environment. This approach is successfully adapted and implemented by [3] using Model Predictive Control (MPC) in combination with an Adaptive Cruise Control (ACC). Ref. [4] suggests to solve the optimal control problem with a strategy of first deriving analytical optimality conditions and evaluating them numerically afterwards. In [5] Pontryagin's Minimum Principle is used to obtain an optimal velocity trajectory. It was concluded that for conventional vehicles, under constant load conditions (e.g. constant road grade), the optimal velocity trajectory subject to a time constraint consists of, full pedal acceleration, followed by a constant velocity part, a coast part and finally a hard deceleration.

Several remarks can be made to the current literature on velocity trajectory optimization. First, optimal velocity trajectories of conventional vehicles are not necessarily optimal for HEVs [6]. Since these vehicles can recover kinetic energy during braking or driving down hill, the above mentioned

Thijs van Keulen, Bram de Jager and Maarten Steinbuch are with the Control Systems Technology, Department of Mechanical Engineering, Eindhoven University of Technology, 5600 Eindhoven, The Netherlands, e-mail: T.A.C.v.Keulen@tue.nl.

Darren Foster is with TNO business unit Automotive. conclusions do not automatically hold. Secondly, considering that under constant load conditions the solution shape consists of, a.o., constant velocity, solving the optimization problem with an equidistant grid has an unnecessary high numerical load. Therefore, data aggregation and velocity trajectory optimization methods that adapt to the route and vehicle characteristics can be beneficial.

The contribution of this paper is a method that solves the velocity optimization problem for HEVs, based upon information from a Global Navigation Satellite-based System (GNSS). Furthermore, it allows the driver to make a balanced choice between fuel economy and traveling time. Assuming a predefined shape of the velocity trajectory allows construction of an optimization problem with relatively low computational effort. Nonlinear programming techniques are explored to solve the optimization problem. The resulting velocity trajectory is used as input for an ACC system, and to estimate the future power trajectory, which enables optimization of the hybrid component use. The benefit of velocity trajectory optimization is indicated with experimental results.

\section{VELOCITY TRAJECTORY OPTIMIZATION}

Determination of the energy-time optimal velocity trajectories requires a combination of route information and vehicle conditions, see Fig. 1. The relevant route information consists of velocity limitations, curvature and road grade. This information can be derived from a Geographic Information System (GIS) in combination with a routeplanner for the destination indicated by the driver. A GNSS with mapmatching algorithms can then be used to locate the vehicle in the map.

The map data could be transferred, e.g. using a CANbus system [7], with a certain horizon. The CAN messages contain information for small parts of the route, and cannot directly be used by the optimization algorithm. The method outlined in this paper accumulates the $h$ messages, and divides a route into $n$ segments with constant velocity limitations and constant road grade as function of the traveled distance. Segments can also be divided by stopping points. This approach requires a rounding of the GIS data and calculation of relevant curvature and road grade, and is further discussed in Section II-A.

Depending on the vehicle operating conditions, electric machine size and battery capacity, velocity trajectories can be determined that minimize the energy use. In Section II$\mathrm{B}$ it is motivated that these solutions have an approximate solution with a fixed solution shape. Algebraic equations are derived that express the equivalent fuel consumption and traveling time as function of cruising and end velocity, and of electric machine and service brake use in one segment, see Section II-C. An optimization problem is obtained by adding up the results of all segments, see Section II-D. It 


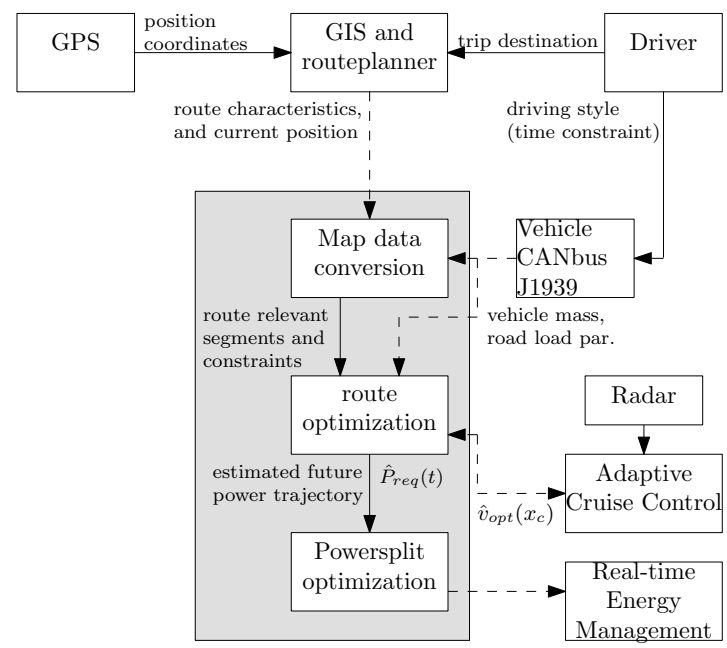

Fig. 1: Topology of velocity trajectory optimization. Legend; $\hat{v}_{\text {opt }}\left(x_{c}\right)$ is the optimized velocity at the actual vehicle position $x_{c}, \hat{P}_{r e q}(t)$ is the estimated power trajectory.

is shown that this optimization problem can be effectively solved using nonlinear programming techniques.

Output of the velocity trajectory optimization is a velocity trajectory and a power trajectory. Perfect tracking of an optimal velocity trajectory will not be possible in actual traffic. Therefore, an ACC system may be employed that consist of an automated cruise control as well as automated adaptation of the vehicle velocity to actual (preceding) traffic [9]. For this, the vehicle can be equipped with a radar, that can provide information about the velocity and distance to a preceding vehicle. In case of a preceding vehicle, the $\mathrm{CC}$ could trigger a recalculation of an optimal velocity trajectory. Besides the velocity trajectory, also a prediction of the future power trajectory is available. This trajectory can be used to optimize the power split trajectory between primary and secondary power converter [10], often referred to as Energy Management Systems (EMS), see [11] for a general overview.

\section{A. Map Data Conversion}

Let us consider a highway trajectory, see Fig. 2 for the topography. From the GIS and routeplanner we obtained CAN messages describing the route ahead. From these messages, the velocity limitation $v_{\max }(h)$, road curvature $\kappa(h)$ and road grade $\beta(h)$ are expressed as function of the traveled distance $l(h)$ with message number $h$.

1) Assumptions on vehicle model: to enable calculation of the optimal velocity trajectory $\hat{v}_{\text {opt }}(x)$, a vehicle model and an estimation of the road load force $\hat{F}_{r l}(h, v)$ is required. Parameters indicated with a hat are estimated values. The road load force depends on the vehicle mass and the route characteristics:

$$
\hat{F}_{r l}(h)=\hat{c}_{0}(h)+\hat{c}_{1}(h) v+\hat{c}_{2}(h) v^{2}
$$

here, $\hat{c}_{0}(h)$ is the drag force independent of velocity $v$ :

$$
\hat{c}_{0}(h)=\hat{F}_{r}(h)+\hat{F}_{g}(h)
$$

with $F_{r}$ the rolling resistance:

$$
\hat{F}_{r}(h)=c_{r} \hat{m} g \cos \beta(h)
$$

in which $c_{r}$ is the rolling resistance coefficient, $g$ is the gravitational constant, $\hat{m}$ is the estimated vehicle mass and $\beta$ is the road grade. The gravitational force is estimated with:

$$
\hat{F}_{g}=\hat{m} g \sin \beta(h) \text {. }
$$

Furthermore, $\hat{c}_{1}$ is the coefficient for drag force linearly depending on velocity. $\hat{c}_{2}(h)$ is the coefficient for the drag force depending on vehicle velocity squared:

$$
\hat{c}_{2}(h)=\frac{1}{2} \rho_{a}(h) A_{f} c_{d}
$$

here, $\rho_{a}$ is the air density, $A_{f}$ is the vehicle frontal area and $c_{d}$ is the aerodynamic coefficient.

It is assumed that the measured relation between the produced engine power and the fuel consumption can be approximated with an affine relation:

$$
\dot{m}_{f}=\dot{m}_{f 0}+\frac{k_{i c e}}{h_{f}} P_{i c e}
$$

here, $\dot{m}_{f}$ is the approximated fuel mass flow, $\dot{m}_{f 0}$ is the fuel mass flow at zero torque, $k_{i c e}$ corresponds to the combustion efficiency and engine internal losses, and $h_{f}$ is the lower heating value of the fuel. The engine power is constrained to the set $P_{i c e} \in\left[P_{\text {drag }}, P_{\text {max }}\right]$, here, $P_{\text {drag }}$ is the engine drag power, and $P_{\max }$ is the maximum available propulsion power. It is known [12] that disregarding the engine rotational velocity dependent ICE losses might lead to suboptimal results.

The electric machine efficiency is approximated as constant and independent of rotational velocity with $\eta_{e m}$ :

$$
P_{b}(t)=\eta_{e m} P_{e m}(t)
$$

in which $P_{b}$ is the electric power, and $P_{e m}$ is the electric machine mechanical power, where we assume that $P_{e m} \in$ $\left[0, \bar{P}_{e m}\right]$. The battery efficiency is quadratic with the power throughput.

$$
P_{s}(t)=P_{b}(t)-P_{b}^{2}(t) \frac{\left.R\right|_{S O C=0.5}}{\left.V_{b a t}^{2}\right|_{S O C=0.5}}
$$

Here, $P_{s}$ is the effective storage power, $R$ is the battery internal resistance at $S O C=0.5$ and $V_{b a t}$ is the voltage of the battery in equilibrium at $S O C=0.5$. The use of the recovered energy, and therefore the average battery efficiency during discharge is governed by the real-time EMS, therefore a constant linear estimation $\eta_{b a t}$ of the battery discharge efficiency is made.

$$
P_{s}(t)=\eta_{b a t} P_{b}(t)
$$

2) Velocity limitations: the maximum legal velocity $v_{\max }$ on the traject is $80 / 3.6 \mathrm{~m} / \mathrm{s}$ for heavy-duty vehicles. The traject in Fig. 2 does not have road curvature. Nevertheless it can be expected, on different routes, that vehicles need to slow down, such that they do not exceed a maximum centrifugal acceleration.

$$
v_{c u r v}(h)<\sqrt{a_{c f g} \kappa(h)}
$$

Here, $v_{\text {curv }}$ is the maximum vehicle cornering velocity, $\kappa(h)$ is the curve radius, and $a_{c f g}$ the maximum centrifugal acceleration for comfortable and save driving. The influence of road banking, which data might be available in a modern 
routeplanner, is omitted for simplicity. The velocity limitation is defined as the minimum of the maximum (legal) velocity $v_{\max }$, the maximum cornering velocity $v_{c u r v}$ and, possibly, the congestion dictated velocity $v_{\text {traffic }}$.

$$
v_{\text {lim }}(h)=\min \left(v_{\max }(h), v_{\text {curv }}(h), v_{\text {traffic }}(h)\right)
$$

Here $v_{\text {lim }}$ is the velocity limitation. Curvature is rounded
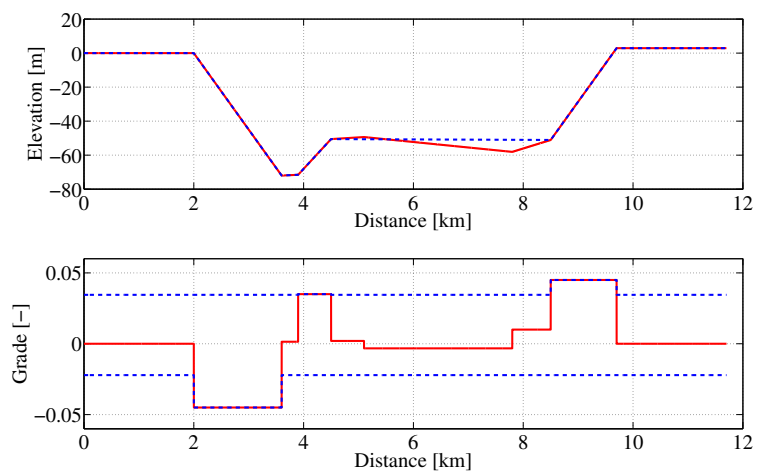

Fig. 2: Route topography for Westerscheldetunnel (N62) from Terneuzen to Elewoutsdijk (the Netherlands).

with an accuracy of $\delta_{\text {curv }} \mathrm{m}^{-1}$. Rounding is required to arrive at segments with a constant velocity limitation. The value of $a_{c f g}$ should be chosen such that even with maximum rounding error, the vehicle will corner safely. Clearly, decreasing $a_{c f g}$ will increase the number of segments required for velocity limitations. Next, road grade segmentation is discussed.

3) Road grades: to arrive at a suitable optimization problem, some assumptions are required. It is assumed here that the velocity dependent loss term $\hat{c}_{1}$, does not change with road grade, unlike [5]. Moreover, the engine efficiency is not depending on the rotational velocity, as in (6). Using these simplifications, gravitational resistance uphill requires a constant amount of fuel, that has to be delivered in any case. Therefore, the road grades can be aggregated, unless the maximum power constraint is reached; so if

$$
\beta_{u p}=\arcsin \left(\frac{\frac{\gamma_{e n g} P_{\max }}{v_{l i m}}-\hat{F}_{r}-\hat{c}_{1} v_{l i m}-\hat{c}_{2} v_{l i m}^{2}}{\hat{m} g}\right)
$$

here, the power discontinuities during gearshifts, and torque limitations, are accounted for by reducing the maximum available power with constant factor $\gamma_{e n g}$, and $\cos \beta=1$ in (3). Moreover, the road grade is rounded with an accuracy of $\delta_{\text {grade }}$, see the lower part of Fig. 2 .

The same kind of reasoning as for uphill driving holds for driving downhill; downhill driving does not change the solution shape, and thus can be aggregated, unless a negative power request is obtained; so if

$$
\beta_{d n}=\arcsin \left(\frac{\frac{P_{\text {drag }}}{\gamma v_{l i m}}+\hat{F}_{r}+\hat{c}_{1} \gamma v_{l i m}+\hat{c}_{2} \gamma v_{l i m}^{2}}{\hat{m} g}\right)
$$

here, $\gamma v_{\text {lim }}$ is the lower velocity constraint.

Road grades $\beta_{d n}<\beta(h)<\beta_{u p}$ are aggregated and averaged. In the example of Fig. 2, the 9 initial segments can be reduced to 7 segments. This results in an approximated elevation, see dashed line in upper part of Fig. 2.

\section{B. Segment Solution}

Using the segments with constant road grade and velocity constraints and based upon assumptions outlined in this section, it is suggested that the velocity trajectory for each segment is described with four phases; max. power acceleration, constant velocity, coast, and max. power deceleration, see Fig. 3. Determination of the velocity trajectory boils down to finding the parameters that describe the length of each phase, subject to the segment velocity and distance constraints, and route time constraint.

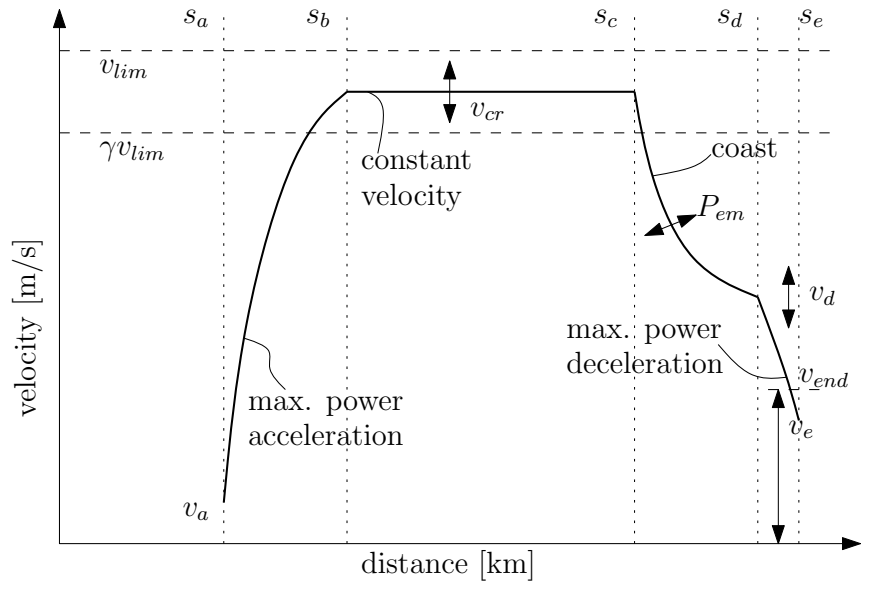

Fig. 3: Velocity trajectory shape for one segment.

The physical motivation for this shape is as follows: as indicated before, $\hat{c}_{0}$ is a velocity independent term. Therefore, provided that the distance of the route is constrained, the energy necessary to overcome $\hat{c}_{0}$ is fixed. Since distance and time are constrained, the energy required to overcome $\hat{c}_{1}$ is also fixed along the route. Therefore, minimizing fuel economy comes down to minimizing the losses that quadratically depend on the velocity $\hat{c}_{2}$, and the loss of kinetic and potential energy in the service brakes and hybrid system. With this reasoning, the time constrained velocity trajectory optimization can be solved by introducing four variables, for each segment, that describe this solution shape: the constant velocity $v_{c r}$, electric machine use $P_{e m}$ during coasting, starting velocity $v_{d}$ of max. power deceleration, and end velocity $v_{e}$ ( $=v_{a}$ of next segment).

Finally, note that a segment could also start with a deceleration, if $v_{a}>v_{c r}$. In that case a coast and max. power deceleration phase is substituted for the max. power acceleration.

\section{Cost Function Construction}

The cost, in terms of fuel consumption and traveling time, for each phase is calculated in this section.

1) Acceleration phase: in [5] it is shown that full throttle accelerations lead to fuel optimal velocity trajectories. Fortunately, real-life driving behavior of heavy-duty vehicles is to use the full power of the vehicle, even for an unloaded vehicle, as was indicated in [13]. Therefore, this approach is 
especially relevant for heavy-duty vehicles. The force at the vehicle wheels is described with:

$$
\hat{F}_{m}(n)=\frac{\gamma_{e n g} P_{\max }}{v}-\hat{F}_{r l}(n) .
$$

The vehicle longitudinal equation of motion becomes:

$$
\frac{d v}{d t}=\frac{\hat{F}_{m}(n)}{\hat{m}_{e}}
$$

here, $\hat{m}_{e}$ is the estimated effective vehicle mass, including the rotational inertia of all rotating parts. Rewriting (15), substituting (14), and integrating from the starting velocity $v_{a}$ to cruising velocity $v_{c r}$ gives the acceleration time:

$$
\left.\Delta t\right|_{t_{a}} ^{t_{b}}=\hat{m}_{e} \int_{v_{a}}^{v_{c r}} \frac{1}{\hat{F}_{m}(n)} d v
$$

This has the solution:

$$
\left.\Delta t\right|_{t_{a}} ^{t_{b}}=\hat{m}_{e} \sum_{q_{a-b}=1}^{3} \frac{R_{q_{a-b}} \ln \left(\frac{v_{c r}-R_{q_{a-b}}}{v_{a}-R_{q_{a-b}}}\right)}{\hat{c}_{0}(n)+2 \hat{c}_{1}(n) R_{q_{a-b}}+3 \hat{c}_{2}(n) R_{q_{a-b}}^{2}}
$$

here $R_{q_{a-b}}$ is the $q_{a-b}$ th root of the cubic equation:

$$
-\gamma_{e n g} P_{\max }+\hat{c}_{0}(n) z+\hat{c}_{1}(n) z^{2}+\hat{c}_{2}(n) z^{3}=0 .
$$

Equation (18) can be solved analytically. The fuel consumption, required during the acceleration part, is expressed algebraically in the start velocity $v_{a}$ and cruise velocity $v_{c r}$ :

$$
m_{f_{a-b}}=\left.\left(\dot{m}_{f 0}+\frac{k_{i c e}}{h_{f}} \gamma_{e n g} P_{\text {max }}\right) \Delta t\right|_{t_{a}} ^{t_{b}} .
$$

The covered distance $\left.\Delta s\right|_{s_{a}} ^{s_{b}}$ is calculated similarly by multiplying (31) $v$. Note that both $\left.\Delta t\right|_{t_{a}} ^{t_{b}}$ and $\left.\Delta s\right|_{s_{a}} ^{s_{b}}$ are functions of the form $x \ln y$. When the end velocity of the previous section is equal to the $v_{c r}$ of the current section, the acceleration part is ignored, and $\left.\Delta t\right|_{t_{a}} ^{t_{b}}$ and $\left.\Delta s\right|_{s_{a}} ^{s_{b}}$ are zero.

2) Coast phase: it is assumed that the vehicle does not change gear during the coast. The electric machine has a constant torque bound $T_{e m}$ at low velocities $v<v_{r m}$ and a constant power bound $P_{e m}$ at high rotational velocities. The force that decelerates the vehicle can then be expressed as:

$$
F_{P_{c-d}}=-\frac{\left(P_{e m}+P_{d r a g}\right)}{v}-\hat{F}_{r l}(n)
$$

for $v \geqslant v_{r m}$, and

$$
F_{T_{c-d}}=-\frac{P_{d r a g}}{v}-\frac{T_{e m} i_{g b} i_{f}}{r_{w}}-\hat{F}_{r l}(n)
$$

for $v<v_{r m}$. Here, $r_{w}$ is the wheel radius, $i_{g b}$ is the actual gearbox ratio, $i_{f}$ is the final drive ratio. The value of $P_{e m}$ is an optimization variable. The deceleration time becomes:

$$
\left.\Delta t\right|_{t_{c}} ^{t_{d}}=\hat{m}_{e} \int_{v_{c r}}^{v_{r m}} \frac{1}{F_{T_{c-d}}} d v+\hat{m}_{e} \int_{v_{r m}}^{v_{d}} \frac{1}{F_{P_{c-d}}} d v .
$$

This has the solutions:

$$
\begin{gathered}
\left.\Delta t\right|_{t_{c}} ^{t_{r m}}= \\
\hat{m}_{e} \sum_{q_{c-r m}=1}^{3} \frac{R_{q_{c-r m}} \ln \left(\frac{v_{r m}-R_{q_{c-r m}}}{v_{c r}-R_{q_{c-r m}}}\right)}{\hat{c}_{0}(n)+2 \hat{c}_{1}(n) R_{q_{c-r m}}+3 \hat{c}_{2}(n) R_{q_{c-r m}}^{2}},
\end{gathered}
$$

$$
\begin{gathered}
\left.\Delta t\right|_{t_{r m}} ^{t_{d}}= \\
\hat{m}_{e} \sum_{q_{r m-d}=1}^{3} \frac{R_{q_{r m-d}} \ln \left(\frac{v_{d}-R_{q_{r m-d}}}{v_{r m}-R_{q_{r m-d}}}\right)}{\hat{c}_{0}(n)+2 \hat{c}_{1}(n) R_{q_{r m-d}}+3 \hat{c}_{2}(n) R_{q_{r m-d}}^{2}},
\end{gathered}
$$

here $R_{q_{c-r m}}$ is the $q_{c-r m}$ th root of the cubic equation:

$$
\left(P_{\text {em }}+P_{\text {drag }}\right)+\hat{c}_{0}(n) z+\hat{c}_{1}(n) z^{2}+\hat{c}_{2}(n) z^{3}=0
$$

and, $R_{q_{r m-d}}$ is the $q_{r m-d}$ th root of the cubic equation:

$$
P_{d r a g}+\left(\frac{T_{e m} i_{g b} i_{f}}{r_{w}}+\hat{c}_{0}(n)\right) z+\hat{c}_{1}(n) z^{2}+\hat{c}_{2}(n) z^{3}=0
$$

The covered distance is calculated similarly by multiplying (22) with $v$. Since we assume that all energy that is recovered is also used during the route, the electric machine efficiency should be squared. The effective recovered energy becomes:

$$
\begin{gathered}
E_{c-d}=\left.\left(-\eta_{e m}^{2} \eta_{b a t} P_{e m}+\left(\eta_{e m}^{2} \eta_{b a t} P_{e m}\right)^{2} \frac{R}{V_{b a t}^{2}}\right) \Delta t\right|_{t_{c}} ^{t_{r m}}+ \\
\left.\left(-\eta_{e m}^{2} \eta_{b a t} \frac{T_{e m} i_{g b} i_{f}}{r_{w}}+\left(\eta_{e m}^{2} \eta_{b a t} \frac{T_{e m} i_{g b} i_{f}}{r_{w}}\right)^{2} \frac{R}{V_{b a t}^{2}}\right) \Delta s\right|_{s_{r m}} ^{s_{d}}
\end{gathered}
$$

The equivalent fuel consumption is:

$$
m_{f_{c-d}}=\frac{E_{c-d}}{h_{f}} \text {. }
$$

3) Deceleration phase: in [5] it is shown that strong deceleration braking leads to fuel optimal velocity trajectories. However, this optimum may not be valid for HEV, since they can recover energy during braking. The following procedure for the deceleration path description is suggested.

The electric machine has a constant torque bound $T_{e m}$ at low velocities $v<v_{r m}$ and a constant power bound $P_{e m}$ at high rotational velocities. The force that decelerates the vehicle can then be expressed as:

$$
F_{P_{d-e}}=-\frac{P_{e m}+P_{d r a g}}{v}-\frac{T_{s e r}}{r_{w}}-\hat{F}_{r l}(n)
$$

for $v \geqslant v_{r m}$, and

$$
F_{T_{d-e}}=-\frac{P_{d r a g}}{v}-\frac{\left(T_{s e r}+T_{e m} i_{g b} i_{f}\right)}{r_{w}}-\hat{F}_{r l}(n)
$$

for $v<v_{r m}$. Here, $T_{s e r}$ is the (maximum) brake torque of the service brakes. It is assumed that there is no change of gear during the deceleration. Note that braking with $T_{e m}<$ $T_{\text {em_max }}$, and $P_{\text {em }}<P_{\text {em_max }}$, is in any case suboptimal, when disregarding the electric machine internal efficiency, and assuming that the battery is not fully charged. The values of $T_{e m}$ and $P_{e m}$ are then known a priori. The deceleration time becomes:

$$
\left.\Delta t\right|_{t_{d}} ^{t_{e}}=\hat{m}_{e} \int_{v_{d}}^{v_{r m}} \frac{1}{F_{T_{d-e}}} d v+\hat{m}_{e} \int_{v_{r m}}^{v_{e}} \frac{1}{F_{P_{d-e}}} d v
$$

This has the solutions:

$$
\begin{aligned}
& \left.\Delta t\right|_{t_{d}} ^{t_{r m}}= \\
& \hat{m}_{e} \sum_{p=1}^{3} \frac{R_{q_{d-r m}} \ln \left(\frac{v_{r m}-R_{q_{d-r m}}}{v_{d}-R_{q_{d-r m}}}\right)}{\hat{c}_{0}(n)+2 \hat{c}_{1}(n) R_{q_{d-r m}}+3 \hat{c}_{2}(n) R_{q_{d-r m}}^{2}},
\end{aligned}
$$




$$
\begin{aligned}
& \left.\Delta t\right|_{t_{r m}} ^{t_{e}}= \\
& \hat{m}_{e} \sum_{r=1}^{3} \frac{R_{q_{r m-e}} \ln \left(\frac{v_{e}-R_{q_{r m-e}}}{v_{r m}-R_{q_{r m-e}}}\right)}{\hat{c}_{0}(n)+2 \hat{c}_{1}(n) R_{q_{r m-e}}+3 \hat{c}_{2}(n) R_{q_{r m-e}}^{2}},
\end{aligned}
$$

here $R_{q_{d-r m}}$ is the $q_{d-r m}$ th root of the cubic equation:

$$
\begin{aligned}
\left(P_{\text {em }}+P_{\text {drag }}\right)+\left(\frac{T_{\text {ser }}}{r_{w}}\right. & \left.+\hat{c}_{0}(n)\right) z \\
& +\hat{c}_{1}(n) z^{2}+\hat{c}_{2}(n) z^{3}=0 .
\end{aligned}
$$

and, $R_{q_{r m-e}}$ is the $q_{r m-e}$ th root of the cubic equation:

$$
\begin{aligned}
P_{d r a g}+\left(\frac{T_{s e r}+T_{e m} i_{g b} i_{f}}{r_{w}}+\hat{c}_{0}(n)\right) z \\
+\hat{c}_{1}(n) z^{2}+\hat{c}_{2}(n) z^{3}=0
\end{aligned}
$$

The covered distance is calculated similarly by multiplying (31) with $v$. Similar to the coast part, the effective recovered energy is calculated by:

$$
\begin{gathered}
E_{d-e}=\left.\left(-\eta_{e m}^{2} \eta_{b a t} \bar{P}_{e m}+\left(\eta_{e m}^{2} \eta_{b a t} \bar{P}_{e m}\right)^{2} \frac{R}{V_{b a t}^{2}}\right) \Delta t\right|_{t_{d}} ^{t_{r m}}+ \\
\left.\left(-\eta_{\text {em }}^{2} \eta_{b a t} \frac{\bar{T}_{e m} i_{g b} i_{f}}{r_{w}}+\left(\eta_{e m}^{2} \eta_{b a t} \frac{\bar{T}_{e m} i_{g b} i_{f}}{r_{w}}\right)^{2} \frac{R}{V_{b a t}^{2}}\right) \Delta s\right|_{s_{r m}} ^{s_{e}}
\end{gathered}
$$

The equivalent fuel consumption is:

$$
m_{f_{d-e}}=\frac{E_{d-e}}{h_{f}} .
$$

4) Constant velocity phase: from the covered distance in the acceleration, coast and deceleration part, follows the distance to be covered with constant velocity:

$$
\left.\Delta s\right|_{s_{b}} ^{s_{c}}=\left(s_{e}-s_{a}\right)-\left.\Delta s\right|_{s_{a}} ^{s_{b}}-\left.\Delta s\right|_{s_{c}} ^{s_{d}}-\left.\Delta s\right|_{s_{d}} ^{s_{e}}
$$

The travel time in this part follows from

$$
\left.\Delta t\right|_{t_{b}} ^{t_{c}}=\frac{\left.\Delta s\right|_{s_{b}} ^{s_{c}}}{v_{c r}}
$$

The power required to overcome the road load forces is:

$$
P_{r e q}=\hat{c}_{0}(n) v_{c r}+\hat{c}_{1}(n) v_{c r}^{2}+\hat{c}_{2}(n) v_{c r}^{3}
$$

Using (6), the fuel consumption on the constant velocity path becomes:

$$
m_{f_{b-c}}=\left.\left(\dot{m}_{f 0}+\frac{k_{i c e} P_{r e q}}{h_{f}}\right) \Delta t\right|_{t_{b}} ^{t_{c}}
$$

5) Segment cost: the equivalent fuel consumption $m_{f}$ and traveling time $t_{n}$ on a segment can both be expressed as a function of the constant velocity $v_{c r}$, electric machine use $P_{e m}$, service brakes appliance velocity $v_{d}$, and end velocity $v_{e}$ :

$$
m_{f_{n}}\left(v_{c r}, P_{e m}, v_{d}, v_{e}\right)=m_{f_{a-b}}+m_{f_{b-c}}+m_{f_{c-d}}+m_{f_{d-e}}
$$

The travel time for the segment becomes:

\section{Optimization Problem Formulation}

In the previous section it is shown that fuel consumption and traveling time on one segment can be expressed, analytically, in vehicle velocity on the constant velocity part, electric machine and service brake use. Therefore, the segment cost can be computed with little computational effort given the four aforementioned parameters. This result is used to construct an optimization problem formulation for the velocity trajectory of the entire route subject to a time constraint.

1) Time constraint as driver input: the driver parameter $\alpha$ is introduced. $\alpha$ allows the driver to make a balanced choice between fast and economic driving. The requested travel time $t_{\text {driver becomes: }}$

$$
t_{\text {driver }}=t_{\text {fast }}-\alpha\left(t_{\text {slow }}-t_{\text {fast }}\right)
$$

in which $\alpha$ is a driver input on the interval $[0,1] . t_{\text {fast }}$ is the shortest traveling time that can be obtained. For calculation of $t_{\text {fast }}$ the upper constraints for every segment are used to solve (43), while for $t_{\text {slow }}$ the lower constraints are used.

2) Optimization over several segments: the cost function is obtained by adding up the segment costs, (42), of all $n$ segments. Solution of this optimization provides the constant velocity $v_{c r}$, electric machine use on the coast part $P_{e m}$, start velocity of max. power deceleration $v_{d}$ and end velocity $v_{e}$ for each segment. Therefore a nonlinear optimization problem can be defined:

$$
\begin{gathered}
\min \\
v_{c r 1}, v_{c r 2}, \ldots, v_{c r n} \\
P_{e m 1}, P_{e m 2}, \ldots, P_{e m n} \\
v_{d 1}, v_{d 2}, \ldots, v_{d n} \\
v_{e 1}, v_{e 2}, \ldots, v_{e n}
\end{gathered}
$$

subject to:

$$
\begin{aligned}
\left(t_{1}+t_{2}+\ldots+t_{n}\right) & \leq t_{\text {driver }} \\
0 & \leq-\left.\Delta s\right|_{s_{b n}} ^{s_{c n}} \\
\gamma v_{\text {limn }} & \leq v_{\text {crn }} \leq v_{\text {limn }} \\
0 & \leq P_{\text {emn }} \leq P_{\text {em_max }} \\
v_{\text {endn }} & \leq v_{d n} \leq v_{\text {crn }} \\
0 & \leq v_{\text {en }} \leq v_{\text {endn }}
\end{aligned}
$$

The problem is solved using the Matlab $^{\circledR}$ function fmincon.

\section{EXPERIMENTAL RESULTS}

The highway route presented in Fig. 2, is used to indicate the fuel benefit of velocity trajectory optimization in a hilly environment. Two situations are evaluated on a chassis dynamometer, with 8 runs each: 1) standard CC use with constant velocity setpoint, 2) an adaptive optimized velocity ACC setpoint with the same time constraint as in case 1.

Fig. 4 shows the velocity trajectories, with dashed the (adaptive) CC setpoints, in solid dark the standard CC results are depicted and in solid gray the optimized results. The equivalent fuel consumption (corrected for SOC deviation) as function of time is depicted in Fig. 5. Typically, the optimized velocity trajectories obtain a fuel consumption benefit due to:

- downhill driving, by decreasing the vehicle velocity in front of the descent, and then to increase the vehicle

$$
t_{n}\left(v_{c r}, P_{e m}, v_{d}, v_{e}\right)=\left.\Delta t\right|_{t_{a}} ^{t_{b}}+\left.\Delta t\right|_{t_{b}} ^{t_{c}}+\left.\Delta t\right|_{t_{c}} ^{t_{d}}+\left.\Delta t\right|_{t_{d}} ^{t_{e}}
$$


velocity during the descent. In this way potential energy is directly converted to kinetic energy. Converting potential energy into kinetic energy proves to be more beneficial then using the hybrid system to store it in the battery.

- a larger average velocity in the first part of the route, to create time for a longer period of regenerative braking/coasting. This longer period outweighs an increase in aerodynamic losses due to a higher constant velocity before the deceleration, in order to obtain an identical traveling time. The average measured fuel consumption benefit is approximately $5 \%$.

\section{CONCLUSIONS AND FUTURE WORK}

A velocity trajectory optimization method is presented that takes advantage of real-life geographical data, is adaptive for vehicle conditions like mass, and computes the optimal velocity trajectory for HEVs, with a computational load that is suitable for real-time implementation. It allows the driver to make a balanced choice between travel time and fuel consumption.

The computed velocity trajectory could be exploited by an adaptive cruise control system. The experimental results show a fuel consumption benefit of 5\% due to velocity trajectory optimization compared to a standard cruise control with constant velocity setpoint. The results show that due to the limited efficiency of the hybrid system, conversion of potential energy into kinetic energy in this situation is preferred over storage in the battery.

Future work will focus on the use of different solvers for the optimization problem, and the integration with the vehicle's real-time energy management.

\section{ACKNOWLEDGMENTS}

The research leading to these results has received funding from the ENIAC Joint Undertaking and from Senter-Novem in the Netherlands under Grant Agreement number 120009, and is part of a more extensive project in the development of advanced energy management control for urban distribution trucks which has been made possible by TNO Business Unit Automotive in cooperation with DAF Trucks NV.

The N.V. Westerscheldetunnel is acknowledged for supplying topographic data of the Westerscheldetunnel route.

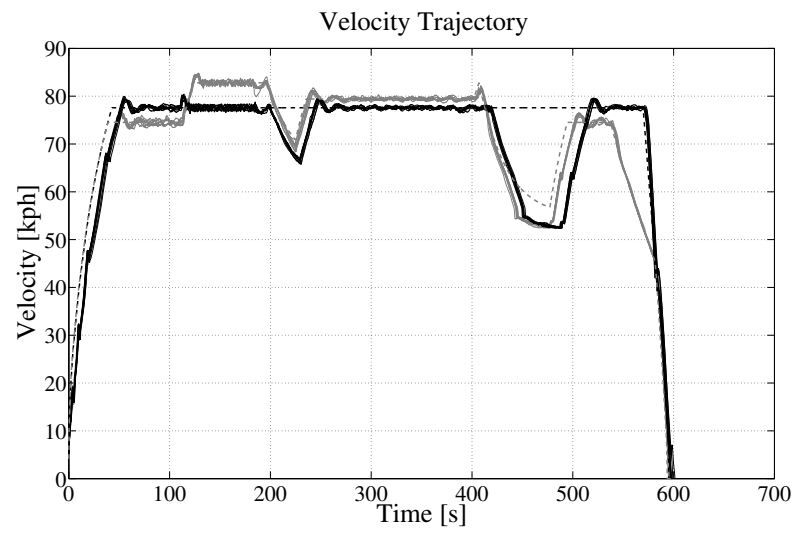

Fig. 4: Velocity trajectories.

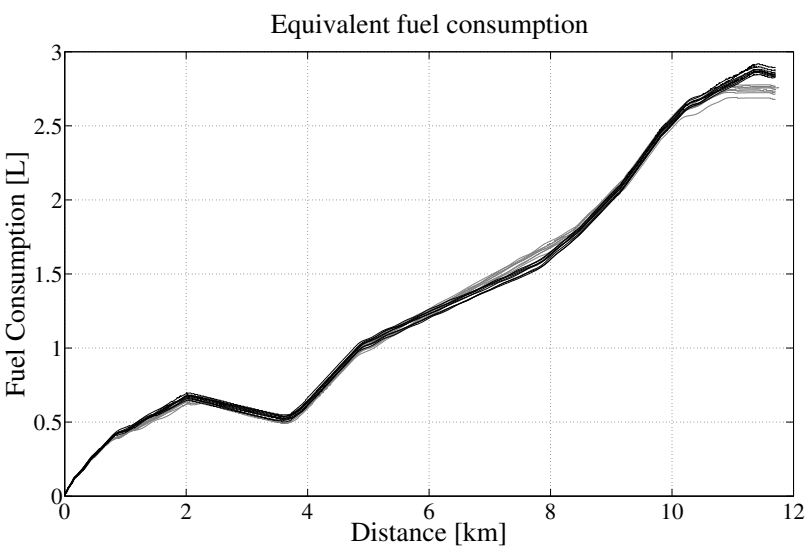

Fig. 5: Equivalent fuel consumption trajectories.

\section{REFERENCES}

[1] T. van Keulen, B. de Jager, M. Steinbuch. Influence of Driver, Route and Vehicle Mass on Hybrid Electric Truck Fuel Economy, AVEC'08 International Symposium on Advanced Vehicle Control, Kobe, Japan, pp. 911-916, October 2008.

[2] V. Monastyrsky, I. Golownykh. Rapid computation of optimal control for vehicles, Transportation Research, Vol. 27B, No. 3, pp. 219-227, 1993.

[3] E. Hellstöm, M. Ivarsson, J. Åslund, L. Nielsen. Look-ahead control for heavy trucks to minimize trip time and fuel consumption, Control Engineering Practice 17, pp. 245-254, July 2008.

[4] B. Passenberg, P. Kock, O. Stursberg. Combined Time and Fuel Optimal Driving of Trucks based on a Hybrid Model, European Control Conference 2009, Budapest, Hungary, pp. 4955-4960, August 23-26, 2009.

[5] A.P. Stoicescu. On Fuel-Optimal Velocity Control of a Motor Vehicle, International Journal of Vehicle Design, Vol. 16, Nos 2/3, pp. 229-256, 1995.

[6] H. Ko, T. Koseki, M. Miyatake. Application of Dynamic Programming to Optimimization of Running Profile of a Train, COMPRAIL 2004, Dresden, Germany, 2004.

[7] L. Beuk, D. Rabel, J. Angenvoort, K. Mezger, M. Perchina. D12.42 Interface and Data Entity Specifications (Final) Part 2 - Data Protocol, IP deliverable of Preventive and Active Safety Applications Integrated Project, March, 15, 2006.

[8] A. Vahidi, A. Stefanopoulou, H. Peng. Recursive least squares with forgetting for online estimation of vehicle mass and road grade: theory and experiments, Vehicle System Dynamics, Vol. 43, No. 1, pp. 31-55, January 2005

[9] T. van Keulen, G.J.L. Naus, B. de Jager, M.J.G. van de Molengraft, E. Aneke, M. Steinbuch. Predictive Cruise Control in Hybrid Electric Vehicles, EVS24, Stavanger, Norway, May 13-18, 2009.

[10] T. van Keulen, B. de Jager, A. Serrarens, M. Steinbuch. Energy Management in Hybrid Electric Trucks Using Route Information, to be printed in Oil \& Gas Science and Technology.

[11] A. Sciarretta, L. Guzzella. Control of Hybrid Electric Vehicles, IEEE Control Systems Magazine, Vol. 27, No. 2, pp. 60-70, April 2007.

[12] B. Saerens, M. Diehl, J. Swevers, E. van den Bulck. Model Predictive Control of Automotive Powertrains - First Experimental Results, Proceedings of the 47th IEEE Conference on Decision and Control, Cancun, Mexico, Dec. 9-11, 2008.

[13] H. Bruneel. Heavy Duty Testing Cycles Development: a New Methology, International Spring Fuels \& Lubricants Meeting \& Exposition, (SAE 2000-01-1890), Paris, France, June 19-20, 2000. 